\title{
Outlining the Prior Infection with SARS-CoV-2 study (PICOV) - preliminary findings on symptoms in nursing home residents and staff.
}

Maria E. Goossens ( $\triangle$ maria.goossens@sciensano.be )

https://orcid.org/0000-0001-7128-1354

Kristof Y. Neven

Sciensano https://orcid.org/0000-0002-1353-0292

Pieter Pannus

Sciensano

Cyril Barbezange

Sciensano

Isabelle Thomas

Sciensano

Steven Van Gucht

Sciensano

Katelijne Dierick

Sciensano

Marie-Noëlle Schmickler

Mensura

Mathieu Verbrugghe

Mensura

Nele Van Loon

Mensura

Kevin K Ariën

Institute of Tropical Medicine: Instituut voor Tropische Geneeskunde

Arnaud Marchant

ULB: Universite Libre de Bruxelles

Stanislas Goriely

ULB: Universite Libre de Bruxelles

Isabelle Desombere

Sciensano 
Keywords: SARS-CoV-2, COVID-19, ILI, ARI, Multicentric, Cohort, Belgium, Antibody, Symptoms, Nursing home

Posted Date: April 5th, 2021

DOI: https://doi.org/10.21203/rs.3.rs-310079/v1

License: (1) This work is licensed under a Creative Commons Attribution 4.0 International License. Read Full License 


\section{Outlining the Prior Infection with SARS-CoV-2 study (PICOV) - preliminary findings on symptoms in nursing home residents and staff}

Maria E Goossens ${ }^{1 * \dagger}$, Kristof $Y$ Neven ${ }^{1 \dagger}$, Pieter Pannus ${ }^{1 \dagger}$, Cyril Barbezange ${ }^{2}$, Isabelle Thomas ${ }^{2}$, Steven Van Gucht ${ }^{2}$, Katelijne Dierick ${ }^{2}$, Marie-Noëlle Schmickler ${ }^{3}$, Mathieu Verbrugghe ${ }^{3}$, Nele Van Loon ${ }^{3}$, Kevin K Ariën ${ }^{4,5}$, Arnaud Marchant ${ }^{6}$, Stanislas Goriely ${ }^{6}$ and Isabelle Desombere ${ }^{2}$

\footnotetext{
${ }^{*}$ Correspondence:

maria.goossens@sciensano.be

${ }^{1}$ SD Epidemiology and Public

Health, Sciensano, Brussels,

Belgium

Full list of author information is available at the end of the article

† Equal contributor
}

\begin{abstract}
Background: COVID-19 has presented itself as one of the most important health concerns of 2020. The geriatric population is arguably hit the hardest by the severe acute respiratory syndrome coronavirus 2 (SARS-CoV-2). The "Prior Infection with SARS-COV-2" (PICOV) study investigates both residents and staff members from nursing homes. The primary aim of the study is to compare the time to occurrence of an influenza-like illness (ILI) or acute respiratory infection (ARI) between participants with a confirmed past SARS-CoV-2 infection and those without an infection. This paper details the study design, sampling scheme, biological measurements, and population characteristics at baseline.
\end{abstract}

Methods: In 26 Belgian nursing homes, all eligible residents and staff members with or without a past SARS-CoV-2 infection (ratio 40/60) were invited to participate. Consent was obtained from 1,375 participants and 1,226 completed the baseline questionnaire. Prevalence of symptoms during a prior SARS-CoV-2 infection was compared between residents and staff members with $\chi^{2}$ statistics.

Results: Nursing home residents (both with and without a prior SARS-CoV-2 infection) systematically reported fewer symptoms than staff members. Moreover, results from prior nasopharyngeal RT-qPCR and baseline serology show that antibody development after a SARS-CoV-2 infection differs between residents and staff members.

Conclusions: We can postulate that disease development and symptoms is different between a geriatric and younger population. Therefore, the occurrence and severity of a future ILI and/or ARI might vary from resident to staff.

Keywords: SARS-CoV-2; COVID-19; ILI; ARI; Multicentric; Cohort; Belgium; Antibody; Symptoms; Nursing home

\section{Background}

At the end of 2019, a novel coronavirus causing acute respiratory disease (COVID19) emerged in the Wuhan region of China and has since led to a worldwide pandemic. The causative agent was named 'severe acute respiratory syndrome coronavirus 2' (SARS-CoV-2) and is closely related to SARS-CoV, which led to an epidemic between 2002 and 2004 [1]. It is estimated that around $40-45 \%$ of all SARS-CoV-2 infections remain asymptomatic [2], for which an dependency on age was observed [3]. Those who do develop COVID-19 present variable clinical out- 
comes, ranging from mild disease with typical symptoms including fever and cough to severe respiratory illness and death $[4,5,6,7]$.

COVID-19 related mortality is strongly age-dependent, with the highest incidence of deaths reported in the geriatric population $[6,8,9]$. Comorbidities related to ageing as well as immunosenescence, i.e. decreased immunological competence due to biological ageing, lie at the basis of this increased risk of death with advanced age [10]. In Belgium, around $10 \%$ of the population aged 65 and older lives in nursing homes where they receive formal long-term care [11], and because of this high concentration of susceptible people in such facilities, more than $60 \%$ of all COVID-19 related deaths between March and June 2020 occurred in nursing home residents [12].

In addition, multiple studies have shown significant co-infection rates of SARSCoV-2 infected patients with viruses causing influenza-like illnesses (ILI) or acute respiratory tract infections (ARI) such as seasonal mild corona viruses, influenza A/B virus, parainfluenzavirus, rhinovirus, bocavirus, human metapneumovirus, and adenovirus $[13,14,15,16]$. As is the case for COVID-19, geriatric people are more at risk for severe clinical forms of these infections. While there are indications that coinfection with e.g. influenza A might increase SARS-CoV-2 viral loads and thereby someone's degree of contagiousness [17], in general little is known about the impact of one infection (previous or current) on the other in terms of susceptibility, immune response and clinical course.

The primary objective of this multi-centre prospective cohort study is to assess whether a confirmed prior infection with SARS-CoV-2 (PICOV) affects the susceptibility to and severity of an ILI and ARI. The aim of the current paper is to describe the PICOV study design, sampling scheme, biological measurements as well as baseline population characteristics.

\section{Methods}

\subsection{Study design}

The PICOV-study is a multi-centre prospective cohort study conducted in 26 nursing homes located in the Belgian regions of Flanders, Wallonia, and Brussels. Both residents as well as staff were eligible to participate. Inclusion of an equal number of participants with and without a previous SARS-CoV-2 infection is targeted.

\subsubsection{Inclusion criteria}

- Aged 18 years or older.

- Having a Belgian National Register Number.

- Being insured by one of the Belgian sickness funds.

- Having the cognitive ability to give consent to participate.

\subsubsection{Exclusion criteria}

- The inability to complete questionnaires in Dutch or French.

- Life-expectancy shorter than the duration of the study.

- Inaccessible veins for simple periphery venipuncture.

- Individuals who will not work or reside at the nursing home for the full duration of the study.

- Individuals who had a previous diagnosis of dementia, having a mini-mental state examination (MMSE) of 18/30 or less. 


\subsection{Objectives of the PICOV study}

The primary objective of the PICOV study is to compare the susceptibility to and the severity of an ILI and ARI in subjects who have had a confirmed prior infection with SARS-CoV-2 compared to those who have not experienced a SARS-CoV-2 infection. The aim of the present manuscript is to describe the study design, sampling scheme, biological measurements, and population characteristics at baseline. Additional study objectives include:

- Determining which viruses are responsible for the ILI or ARI incidences throughout the study.

- Evaluating whether COVID-19 convalescent participants are protected against reinfection with SARS-CoV-2 and identifying serological markers that are associated with protection.

- Assessing the influence of a previous SARS-CoV-2 infection on the immunological response to influenza vaccination.

\subsection{Study flow}

\subsubsection{Recruitment}

Eligible staff members and residents with a documented previous SARS-CoV-2 infection were invited to participate. A serological screening with an antibody rapid diagnostic test (RDT) was performed for those who never received a positive RTqPCR or serological test. Antibody RDTs included the CORIS COVID-19 Ab Rapid Test (CORIS BioConcept, Gembloux, Belgium), COVID-PRESTO ${ }^{\circledR}$ COVID-19 IgG/IgM Rapid Test (AAZ-LMB, Boulogne-Billancourt, France), and the quickZen COVID-19 IgG/IgM Rapid Test (Zentech, Liège, Belgium). Those with a positive RDT result were also invited to participate. Next, approximately $20 \%$ more staff and residents without a documented SARS-CoV-2 infection or positive antibody RDT result were invited and enrolled in the study in order to compensate for asymptomatic infections and false-negative antibody RDT results to reach our target of $50 \%$ with and $50 \%$ without a past SARS-CoV-2 infection. In nursing homes which reported that over $50 \%$ of their residents and staff ever had a positive SARS-CoV-2 RT-qPCR test, no prior RDT screening was done and all eligible participants were immediately invited to participate. Dates and results of previous lung CT-scans, RT-qPCR tests and/or antibody tests were collected by the study nurses.

\subsubsection{Sample collection and biological measurements}

Biological samples, including nasopharyngeal swabs, saliva, and blood, were collected from all participants at baseline and at the end of the study. Figure 2 summarises which samples are collected at each study visit. Participants who were vaccinated against seasonal flu had an extra sampling visit, two weeks after vaccination. The flu vaccines which were administered during the study period included Vaxigrip Tetra (Sanofi S.A., Gentilly, France) and Influvac Tetra (Abbot Biologicals B.V., Olst, The Netherlands). In addition, study participants presenting with symptoms of an ILI or ARI had two extra sampling visits: one within five days of symptom onset and another two to three weeks after symptom onset during the attenuation of their illness. 
Nasopharyngeal swabs (stored in universal transfer medium - UTM ${ }^{\circledR}$ : Viral Transport [Copan, California, USA]) were collected to detect an ongoing SARSCoV-2 infection by RT-qPCR. Serum ( 8 mL CAT Serum Separator Clot Activator Vacuette ${ }^{\circledR}$ Tubes, Greiner Bio-One, Kremsmünster, Austria) was collected for serological measurements. Heparinised blood (x $10 \mathrm{~mL}$ plastic BD Vacutainer Lithium Heparine tubes (BD, New Jersey, USA) was collected for plasma and peripheral blood mono-nuclear cell (PBMC) isolation for cellular immunity studies. Oral fluid (Oracol Saliva System, Malvern Medical Developments Ltd, Worcester, UK) was collected to study mucosal immunity.

\subsection{Questionnaires}

Participants completed questionnaires at baseline, at the end of the study and in the case an ILI or ARI occurs. Staff members received a personal link to fill out the questionnaires themselves on-line, while residents were assisted by the study nurses.

\subsubsection{Baseline}

The questionnaire at baseline provides detailed basic information about participant age, gender, self-reported weight and height, ethnicity, smoking status, and physical activity. Medication use, prior SARS-CoV-2-test results (i.e. RT-qPCR, lung CTscan, and/or serology), vaccination status (i.e. pneumococcal vaccination in the past five years and influenza vaccination in the past year) are also recorded. In addition, the profession of staff members as well as the Katz-scale and MMSE-score of residents were also recorded.

Finally, participants were required to answer questions regarding any previous COVID-19 episode or influenza-like symptoms. These questions recorded the symptoms, their duration, the impact of COVID-19 on their daily activities and the severity of the illness as approximated by hospitalisation and/or ventilation requirements.

Standardised questionnaires were also provided and included a standardised measure for the health status: EQ-5D five-level (EQ-5D-5L) by EuroQol [18]. For residents, frailty was assessed via the 9-point Clinical Frailty Scale [19].

\subsubsection{End of study}

The questionnaire at the end of the study collects, besides current weight, smoking status and physical activity of the participants, information on COVID-19 episodes or influenza-like symptoms that occurred during the study period. Similar to the baseline questionnaire, (duration of) symptoms, impact of COVID-19 on daily activities and severity of illness are recorded.

The standardised questionnaires are collected once more: EQ-5D-5L for both staff and residents, and the 9-point Clinical Frailty Scale for only the residents.

\subsection{Biological measurements at baseline}

Nasopharyngeal swabs tested for the presence of SARS-CoV-2 RNA with an RTqPCR targeting the E gene, according to the protocol by Corman et al. [20]. The Ct cut-off for positivity was 40 .

The Wantai SARS-CoV-2 Ab ELISA (cat n ${ }^{\circ}$ WS-1096; Beijing Wantai Biological Pharmacy Enterprise Co. Ltd., China) detects anti-receptor binding domain (RBD) 
IgG, IgA and IgM simultaneously in serum. In house and reported validations show a test specificity of $99.6 \%$ and a sensitivity of $100 \%$ at 14 days post-clinical illness onset $[21,22,23]$.

\subsection{Statistical analysis}

Database management and data analysis were performed with the $\mathrm{R}$ software (version 4.0.3.). Descriptive statistics are used to describe the baseline characteristics. Mean \pm standard deviation $( \pm \mathrm{SD})$ is given for continuous variables and the proportion (\%) for categorical variables. Normality of data distributions was tested with the Shapiro-Wilk statistics.

We assessed the proportions of categorical variables ( $\chi^{2}$-statistics). The MannWhitney test was performed for the analysis on number of symptoms, as the data was not normally distributed.

\section{Results of baseline characteristics}

\subsection{Recruitment and sample size}

Recruitment of participants started on September $24^{\text {th }}, 2020$, and ended on December $8^{\text {th }}, 2020$. A total of 1,375 participants initially provided informed consent to participate (Figure 1). 116 individuals (8.4\%) withdrew from the study resulting in a final sample size of 1,226 participants. Baseline sampling was completed on December $\left.8^{8}, 2020\right)$ and sampling after flu vaccination was completed on January $\left.25^{\text {th }}, 2021\right)$.

\subsection{Population characteristics}

Sociodemographic characteristics of all participants are presented in Table 1. Between March and August 2020, most participants were routinely tested by RT-qPCR with a nasopharyngeal swab $(\mathrm{n}=1,197$ [97.6\%]) and a minority had their blood drawn for a SARS-CoV-2 antibody test $(\mathrm{n}=200$ [16.3\%]). At baseline, 18 participants $(1.5 \%)$ tested SARS-CoV-2 positive by RT-qPCR. The current study includes 374 residents $(30.5 \%)$ and 852 staff members $(69.5 \%)$.

\subsubsection{Residents}

The 374 residents had an average age of 81.9 years $( \pm 10.7)$ and a mean BMI of $25.5 \mathrm{~kg} / \mathrm{m}^{2}( \pm 5.3)$. More than two-thirds was female $(64.4 \%)$, and $99.2 \%$ was of European descent. Most residents never smoked (72.2\%).

\subsubsection{Staff}

Staff members had an average age of 44.2 years $( \pm 11.5)$ and mean BMI of 26.1 $\mathrm{kg} / \mathrm{m}^{2}( \pm 4.8)$. The majority was female $(85.3 \%)$ and 747 were of European descent (87.7\%). 619 (72.7\%) staff members reported to have never smoked. Nurses $(23.2 \%)$ and nursing assistants (29.7\%) were the most included professions (Table 3). In total, $52(6.1 \%)$ staff members worked in specialised COVID departments within their respective nursing homes. 27 of them indicated to have worked solely in these (temporary) departments. 


\subsection{SARS-CoV-2 history at baseline}

Previous nasopharyngeal RT-qPCR and serology data indicated that 445 participants reported to have had a positive SARS-CoV-2 RT-qPCR or serology test between March and August 2020, while 781 reported no or only negative RT-qPCR or serology tests (Table 2). Taking serology at baseline into account, 586 participants (47.8\%) had neither a positive SARS-CoV-2 RT-qPCR nor a positive anti-RBD IgG antibody test and are therefore categorised as 'no past SARS-CoV-2 infection'. The remaining 640 participants $(52.2 \%)$ are categorised as 'past SARS-CoV-2 infection'. Of the latter group, $61(9.5 \%)$ had a positive RT-qPCR but no detectable antibodies at baseline, $182(28.4 \%)$ never had a positive RT-qPCR but did have detectable antibodies at baseline, and $397(62.0 \%)$ had a previous positive RT-qPCR result and detectable antibodies at baseline.

As expected per our study protocol, the proportion of participant with 'no past SARS-CoV-2 infection' did not differ between residents and staff members $(52.4 \%$ and $47.4 \%$ respectively; $\chi^{2}=0.41, \mathrm{p}=0.52$ ). For participants with a 'past SARSCoV-2 infection', fewer residents had detectable Ab after a positive RT-qPCR test than staff members $\left(24.4 \%\right.$ and $35.5 \%$, respectively; $\left.\chi^{2}=12.5, \mathrm{p}=0.0004\right)$. Conversely, more residents than staff members had detectable $\mathrm{Ab}$ without a previous positive RT-qPCR (24.4\% and $14.2 \%$, respectively; $\left.\chi^{2}=12.8, \mathrm{p}=0.0004\right)$. The proportion of participants with a previous positive RT-qPCR and no detectable $\mathrm{Ab}$ at baseline did not differ between residents and staff members $(2.1 \%$ and $4.6 \%$, respectively; $\chi^{2}=0.0, \mathrm{p}=0.99$ ).

\subsection{Symptoms from March to August 2020}

Participants with a past SARS-CoV-2 infection reported significantly more symptoms than those without an infection $(\mathrm{p}<0.0001)$, at $5.5( \pm 3.9)$ and $3.0( \pm 2.3)$ symptoms, respectively (Figure 3 ). This effect was the strongest in the staff members, as those with a past infection had $6.1( \pm 4.0)$ symptoms compared to $3.1( \pm 2.2)$ symptoms for staff members without an infection $(\mathrm{p}<0.0001)$. In residents, a similar effect was observed as the number of symptoms during a past SARS-CoV-2 infection was higher compared to those without an infection (3.1 vs 1.9 symptoms, $\mathrm{p}=0.0155)$.

\subsubsection{Residents}

From March until August 2020, 188 residents had a 'past SARS-CoV-2 infection', of whom the most (111 [59.0\%]) reported to have had no symptoms during their prior infection (Table 4). Fatigue (40 [21.3\%]) and fever (39 [20.7\%]) were the most and second most reported symptoms, respectively.

For the 183 residents with 'no past SARS-CoV-2 infection', 161 reported to have had no symptoms (88.0\%; Table 5).

\subsubsection{Staff}

Of the 452 staff members with a 'past SARS-CoV-2 infection', the majority indicated to have had fatigue (256 [56.6\%]; Table 4). Nearly half of the staff reported symptoms of ageusia and/or anosmia (202 [44.7\%]) or a headache (195 [43.1\%]). 127 (28.1\%) staff members indicated to have had no symptoms during their infection. 
291 of the $403(72.2 \%)$ staff members with 'no past SARS-CoV-2 infection' reported to have had no symptoms (Table 5). Only a few reported to have had a headache $(56[13.9 \%])$, a sore throat $(56[12.9 \%])$, or fatigue $(50[12.4 \%])$.

\subsection{Persisting symptoms at baseline sampling}

Participants with a past SARS-CoV-2 infection were asked whether they experienced any more symptoms at baseline sampling (approximately one to ten months after infection; Table 6). Only 8 (4.3\%) of the 188 residents with a past SARS-CoV2 infection indicated to have symptoms at baseline sampling, such as coughing (4 $[50.0 \%])$, fatigue and dyspnea $(2[25.0 \%])$. Of the staff members with a prior infection, $86(19.2 \%)$ indicated to have symptoms at baseline. Most indicated to have fatigue (48 [55.8\%]), ageusia and/or anosmia (34 [39.5\%]), or dyspnea (31 [36.0\%]).

\section{Discussion}

The PICOV study is a prospective multi-centre study designed to investigate the effects of a prior SARS-CoV-2 infection on the occurrence and severity of ILI and ARI among residents and staff members of nursing homes. At the moment of publication, enrolment, baseline sampling, and follow-up sampling after vaccination have been completed. Comparing the symptoms between nursing home residents and staff members showed some interesting differences. On the one hand, residents have a higher proportion of asymptomatic cases (i.e. negative prior RT-qPCR results and detectable $\mathrm{Ab}$ at baseline) than staff members. On the other hand, staff members had a higher percentage of prior positive RT-qPCR swabs. Moreover, staff members with a past SARS-CoV-2 infection systematically report more symptoms than either residents with a past infection and staff members without a past infection. In contrast, residents with a past SARS-CoV-2 infection reported slightly more symptoms than residents whitout a past infection, albeit statistically not significant.

Fatigue and fever were the most prevalent self-reported symptoms during a 'past SARS-CoV-2 infection', accounting for over a third of nursing home residents. A recent systematic review by Neumann-Podczaska and colleagues described symptom data of 1,285 older people (aged 60 years and over) from 20 studies [24]. The authors reported that fever and cough were the most common symptoms at $83.6 \%$ and $62.7 \%$, respectively. Although fever was the second highest reported symptom among nursing home residents in our study, only $28.1 \%$ reported a fever during their COVID-19 episode. In contrast to these earlier findings [24], our nursing home residents reported less coughing ( $18.1 \%$ in PICOV compared to $62.7 \%$ by NeumannPodczaska). The reasons for these differences are not clear, but could firstly be attributed to memory recall errors as this data is collected retrospectively [25]. Secondly, the study population is different, as our data includes only current residents at nursing homes that survived their SARS-CoV-2 infection. This is in contrast with the systematic review [24], where half of the participants were hospitalised and a fifth of the total population died. Finally, our findings are more in line with findings from care homes in the UK [26], as the authors report a more comparable proportion of fever and coughing.

More than half (56.6\%) of the nursing home staff members with a past SARS-CoV-

2 infection reported to be fatigued during their COVID-19 episode, which makes 
this the most prevalent symptom. This incidence is in line with studies in Italian $(47.6 \%)$ and Swedish $(65.0 \%)$ healthcare workers (HCW) in hospitals [27, 28]. The prevalence of most of our symptoms are consistent with the observations made by Rudberg et al. [28]. Moreover, while a quarter of the HCW in Sweden [28] and nearly a third of the Italian HCW [27] documented dyspnoea as a symptom, $27.8 \%$ of our staff members mentioned dyspnoea during their COVID-19 illness. Although the prevalence of symptoms in the present cohort is comparable to those observed in HCW, we should note some key differences between these populations. First and foremost, both the Swedish and Italian study investigated HCW in hospitals, which is fundamentally different from a nursing home setting. Moreover, we enrolled fewer nurses and physicians and more nonmedical support staff such as administrative, support, and kitchen staff.

In the PICOV cohort, asymptomatic SARS-CoV-2 infections (i.e. received no or negative RT-qPCR nasal swabs and a positive Ab test) occur more frequent in residents than in staff members. This is in line with recent observations by Mori et al. [3], who noted three times as many asymptomatic cases in older people (60 years and over) than in young people (18 - 60 years old) with a confirmed SARS-CoV-2 infection. This is corroborated by the symptom data, as residents systematically reported fewer symptoms than staff members in PICOV. Another symptom that was reported less frequently among the geriatric population as compared to the staff was ageusia and/or anosmia. This is in agreement with previous findings [29, 30] and could be attributed to normal ageing, as taste and smell tend to subside with age [31].

A third of the staff members reported persistent symptoms at baseline, with fatigue, ageusia and/or anosmia, and dyspnoea being the most prevalent, which corroborates previous findings [32, 33]. In contrast, only a tenth of the residents indicated any persistent symptom at baseline. Most of them indicated to have a cough, fatigue, or dyspnoea. In both residents and staff members, fatigue was still present a few months after their SARS-CoV-2 infection. In 2009, a follow-up study of 233 SARS survivors in Hong Kong observed that $40 \%$ of the responders had a chronic fatigue problem at their four-year follow-up [34]. Interestingly, the geriatric population reported fewer symptoms during their SARS-CoV-2 infection and fewer persisting symptoms 1-10 months afterwards. In summary, nearly three quarters of the PICOV participants indicated to have no more nor additional symptoms one to ten months after symptom onset, which is in line with other non-hospitalised COVID-19 individuals [35].

Our study has several strengths. Firstly, we recruited a representative sample of both staff members and residents of nursing homes across Belgium. As such, our research population includes participants 18 to 102 years old, providing a broader range of ages compared to other published studies. Secondly, the participants are followed closely and sampled frequently in the case of ILI or ARI, allowing us to capture the development of SARS-CoV-2 Ab already early on during an infection. Thirdly, additional samples are collected at the various sampling moments, which allows for additional measurements for immune response monitoring.

We also acknowledge some limitations. First of all, the symptom registration of the COVID-19 episode occurs one to ten months after symptom onset and is therefore 
subjected to recall bias [36]. Nevertheless the authors proposed that patients tend to recall symptoms better than health-related quality of life. Another shortcoming is that we are unable to capture those individuals with asymptomatic SARS-CoV-2 infections for the duration of the PICOV study with our case definition.

\section{Conclusion}

Nursing home residents (both with and without a prior SARS-CoV-2 infection) systematically reported fewer symptoms than staff members. Moreover, results from prior nasopharyngeal RT-qPCR and baseline serology show that antibody development after a SARS-CoV-2 infection differs between residents and staff members. As such, we can postulate that disease development and symptoms are different between a geriatric and younger population. Therefore, the influence of a prior SARS-CoV-2 infection on the occurrence and severity of a future ILI and/or ARI might differ between residents and staff.

\section{List of Abbreviations}

- ARI: acute respiratory tract infection

- EQ-5D-5L: EQ-5D five-level

- HCW: healthcare workers

- ILI: influenza-like illness

- MMSE: mini-mental state examination

- PBMC: peripheral blood mono-nuclear cell

- PICOV: prior infection with SARS-CoV-2

- RBD: receptor binding domain

- RDT: rapid diagnostic test

- SARS-CoV-2: severe acute respiratory syndrome coronavirus 2

\section{Declarations}

Ethics approval and consent to participate

The study was approved by the Ethics Committee of the Hôpital Erasme in Brussels, Belgium (reference: P2020/424/ B4062020000134) and is registered at ClinicalTrials.gov (reference: NCT04527614).

Consent for publication

Not applicable.

Availability of data and material

The data sets analysed during the current study are available from the corresponding author on reasonable request.

Competing interests

The authors declare that they have no competing interests.

Funding

MEG obtained funding from The Belgian Federal Government.

Author's contributions

MEG conceptualised, designed, and coordinates the PICOV-study, and wrote the first draft of the manuscript. KYN helped to draft the manuscript and performed the statistical analyses and data visualisation. PP helped to draft the manuscript and aided in the data visualisation and coordination of the study. CB, IT, and ID performed the RT-qPCR measurements and helped design the study. MNS, MV, and NVL helped in the design and conceptualisation of PICOV, and are responsible for the study nurses. KKA, AM, and SG were also involved in the study design, and performed PBMC isolations. All authors critically reviewed and approved the manuscript. 
Acknowledgements

We thank the members of the steering committee for their valuable feedback on the manuscript: Van Damme $P$ (University of Antwerp), Leroux-Roels G and Petrovic M (Ghent University Hospital), and Hulstaert F and Ricour C (Belgian Health Care Knowledge Centre). In addition, we thank the lab members for extracting the PBMC: Bartholomeeusen K, Ceulemans A, Coppens S, De Caluwé L, Escandell ML, Heyndrickx L, Michiels L, and Willems B (Institute of Tropical Medicine); Fdillate I, Van Eycken R, Hamouda A, Jurion F, Rodeghiero C, Vande Kerckhove S, and Francote A (Sciensano); Brauns E, Nguyen M, Thomas S, Olislagers V, Ottou MA, McKinven K, Ballieu F, Nana Tchouta VG, and Vu Duc I (Université libre de Bruxelles). A special thank goes out to the study nurses Degraeve S, Delaere M, Louvrier I, Massez K, Matia Sangrador N, and Serré J. Lastly, we also want to thank the coordinating staff and directorate of the nursing homes and all the participants of the PICOV study for their efforts and participation.

\section{Author details}

${ }^{1}$ SD Epidemiology and Public Health, Sciensano, Brussels, Belgium. ${ }^{2}$ SD Infectious Diseases in Humans, Sciensano, Brussels, Belgium. ${ }^{3}$ Mensura Occupational Health Service, Antwerp, Belgium. ${ }^{4}$ Virology Unit, Department of Biomedical Sciences, Institute of Tropical Medicine, Antwerp, Belgium. ${ }^{5}$ Department of Biomedical Sciences, University of Antwerp, Antwerp, Belgium. ${ }^{6}$ Institute for Medical Immunology, Université libre de Bruxelles, Charleroi, Belgium.

\section{References}

1. Zhou, P., Yang, X.-L., Wang, X.-G., Hu, B., Zhang, L., Zhang, W., Si, H.-R., Zhu, Y., Li, B., Huang, C.-L., Chen, H.-D., Chen, J., Luo, Y., Guo, H., Jiang, R.-D., Liu, M.-Q., Chen, Y., Shen, X.-R., Wang, X., Zheng, X.-S., Zhao, K., Chen, Q.-J., Deng, F., Liu, L.-L., Yan, B., Zhan, F.-X., Wang, Y.-Y., Xiao, G.-F., Shi, Z.-L.: A pneumonia outbreak associated with a new coronavirus of probable bat origin. Nature 579(7798), 270-273 (2020). doi:10.1038/s41586-020-2012-7. Number: 7798 Publisher: Nature Publishing Group. Accessed 2021-01-11

2. Oran, D.P., Topol, E.J.: Prevalence of Asymptomatic SARS-CoV-2 Infection : A Narrative Review. Annals of Internal Medicine 173(5), 362-367 (2020). doi:10.7326/M20-3012

3. Mori, H., Obinata, H., Murakami, W., Tatsuya, K., Sasaki, H., Miyake, Y., Taniguchi, Y., Ota, S., Yamaga, M., Suyama, Y., Tamura, K.: Comparison of COVID-19 disease between young and elderly patients: Hidden viral shedding of COVID-19. Journal of Infection and Chemotherapy 27(1), 70-75 (2021). doi:10.1016/j.jiac.2020.09.003. Accessed 2021-01-26

4. Wu, D., Wu, T., Liu, Q., Yang, Z.: The SARS-CoV-2 outbreak: What we know. International Journal of Infectious Diseases 94, 44-48 (2020). doi:10.1016/j.ijid.2020.03.004. Accessed 2021-01-11

5. Chen, N., Zhou, M., Dong, X., Qu, J., Gong, F., Han, Y., Qiu, Y., Wang, J., Liu, Y., Wei, Y., Xia, J., Yu, T. Zhang, X., Zhang, L.: Epidemiological and clinical characteristics of 99 cases of 2019 novel coronavirus pneumonia in Wuhan, China: a descriptive study. The Lancet 395(10223), 507-513 (2020). doi:10.1016/S0140-6736(20)30211-7. Accessed 2021-01-11

6. O'Driscoll, M., Ribeiro Dos Santos, G., Wang, L., Cummings, D.A.T., Azman, A.S., Paireau, J., Fontanet, A., Cauchemez, S., Salje, H.: Age-specific mortality and immunity patterns of SARS-CoV-2. Nature, 1-6 (2020). doi:10.1038/s41586-020-2918-0. Publisher: Nature Publishing Group. Accessed 2021-01-11

7. Jordan, R.E., Adab, P., Cheng, K.K.: Covid-19: risk factors for severe disease and death. BMJ (Clinical research ed.) 368, 1198 (2020). doi:10.1136/bmj.m1198

8. Hardy, O.J., Dubourg, D., Bourguignon, M., Dellicour, S., Eggerickx, T., Gilbert, M., Sanderson, J.-P., Scohy, A., Vandael, E., Decroly, J.-M.: A world apart: levels and factors of excess mortality due to COVID-19 in care homes. The case of Wallonia - Belgium. medRxiv, 2020-082920183210 (2020). doi:10.1101/2020.08.29.20183210. Publisher: Cold Spring Harbor Laboratory Press. Accessed 2021-01-11

9. Weiss, P., Murdoch, D.R.: Clinical course and mortality risk of severe COVID-19. Lancet (London, England) 395(10229), 1014-1015 (2020). doi:10.1016/S0140-6736(20)30633-4

10. Chen, Y., Klein, S.L., Garibaldi, B.T., Li, H., Wu, C., Osevala, N.M., Li, T., Margolick, J.B., Pawelec, G., Leng, S.X.: Aging in COVID-19: Vulnerability, immunity and intervention. Ageing Research Reviews 65, 101205 (2021). doi:10.1016/j.arr.2020.101205

11. Care for the elderly - For a Healthy Belgium. https://www.healthybelgium.be/en/health-system-performanceassessment/specific-domains/care-for-the-elderly Accessed 2021-01-11

12. Molenberghs, G., Faes, C., Verbeeck, J., Deboosere, P., Abrams, S., Willem, L., Aerts, J., Theeten, H., Devleesschauwer, B., Sierra, N.B., Renard, F., Herzog, S., Lusyne, P., Heyden, J.V.d., Oyen, H.V., Damme, P.V., Hens, N.: Belgian COVID-19 Mortality, Excess Deaths, Number of Deaths per Million, and Infection Fatality Rates (9 March - 28 June 2020). medRxiv, 2020-062020136234 (2020). doi:10.1101/2020.06.20.20136234. Publisher: Cold Spring Harbor Laboratory Press. Accessed 2021-02-19

13. Wang, M., Wu, Q., Xu, W., Qiao, B., Wang, J., Zheng, H., Jiang, S., Mei, J., Wu, Z., Deng, Y., Zhou, F., Wu, W., Zhang, Y., Lv, Z., Huang, J., Guo, X., Feng, L., Xia, Z., Li, D., Xu, Z., Liu, T., Zhang, P., Tong, Y., Li, Y.: Clinical diagnosis of 8274 samples with 2019-novel coronavirus in Wuhan. medRxiv, 2020-021220022327 (2020). doi:10.1101/2020.02.12.20022327. Publisher: Cold Spring Harbor Laboratory Press. Accessed 2021-01-11

14. Lin, D., Liu, L., Zhang, M., Hu, Y., Yang, Q., Guo, J., Guo, Y., Dai, Y., Xu, Y., Cai, Y., Chen, X., Zhang, Z., Huang, K.: Co-infections of SARS-CoV-2 with multiple common respiratory pathogens in infected patients. Science China. Life Sciences 63(4), 606-609 (2020). doi:10.1007/s11427-020-1668-5

15. Cuadrado-Payán, E., Montagud-Marrahi, E., Torres-Elorza, M., Bodro, M., Blasco, M., Poch, E., Soriano, A., Piñeiro, G.J.: SARS-CoV-2 and influenza virus co-infection. Lancet (London, England) 395(10236), 84 (2020). doi:10.1016/S0140-6736(20)31052-7 
16. Hashemi, S.A., Safamanesh, S., Ghasemzadeh-Moghaddam, H., Ghafouri, M., Azimian, A.: High prevalence of SARS-CoV-2 and influenza A virus (H1N1) coinfection in dead patients in Northeastern Iran. Journal of Medical Virology 93(2), 1008-1012 (2021). doi:10.1002/jmv.26364

17. Bai, L., Zhao, Y., Dong, J., Liang, S., Guo, M., Liu, X., Wang, X., Huang, Z., Sun, X., Zhang, Z., Dong, L., Liu, Q., Zheng, Y., Niu, D., Xiang, M., Song, K., Ye, J., Zheng, W., Tang, Z., Tang, M., Zhou, Y., Shen, C., Dai, M., Zhou, L., Chen, Y., Yan, H., Lan, K., Xu, K.: Co-infection of influenza A virus enhances SARS-CoV-2 infectivity. bioRxiv, 2020-1014335893 (2020). doi:10.1101/2020.10.14.335893. Publisher: Cold Spring Harbor Laboratory Section: New Results. Accessed 2021-01-21

18. Herdman, M., Gudex, C., Lloyd, A., Janssen, M., Kind, P., Parkin, D., Bonsel, G., Badia, X.: Development and preliminary testing of the new five-level version of EQ-5D (EQ-5D-5L). Quality of Life Research: An International Journal of Quality of Life Aspects of Treatment, Care and Rehabilitation 20(10), 1727-1736 (2011). doi:10.1007/s11136-011-9903-x

19. Rockwood, K., Theou, O.: Using the Clinical Frailty Scale in Allocating Scarce Health Care Resources. Canadian Geriatrics Journal 23(3), 210-215 (2020). doi:10.5770/cgj.23.463. Accessed 2021-01-12

20. Corman, V.M., Landt, O., Kaiser, M., Molenkamp, R., Meijer, A., Chu, D.K., Bleicker, T., Brünink, S., Schneider, J., Schmidt, M.L., Mulders, D.G., Haagmans, B.L., Veer, B.v.d., Brink, S.v.d., Wijsman, L., Goderski, G., Romette, J.-L., Ellis, J., Zambon, M., Peiris, M., Goossens, H., Reusken, C., Koopmans, M.P., Drosten, C.: Detection of 2019 novel coronavirus (2019-nCoV) by real-time RT-PCR. Eurosurveillance 25(3), 2000045 (2020). doi:10.2807/1560-7917.ES.2020.25.3.2000045. Publisher: European Centre for Disease Prevention and Control. Accessed 2021-02-11

21. Lassaunière, R., Frische, A., Harboe, Z.B., Nielsen, A.C.Y., Fomsgaard, A., Krogfelt, K.A., Jørgensen, C.S.: Evaluation of nine commercial SARS-CoV-2 immunoassays. medRxiv, 2020-040920056325 (2020). doi:10.1101/2020.04.09.20056325. Publisher: Cold Spring Harbor Laboratory Press. Accessed 2021-02-11

22. GeurtsvanKessel, C.H., Okba, N.M.A., Igloi, Z., Embregts, C.W.E., Laksono, B.M., Leijten, L., Rahamat-Langendoen, J., Akker, J.P.C.v.d., Kampen, J.J.A.v., Eijk, A.A.v.d., Binnendijk, R.S.v., Haagmans, B., Koopmans, M.: Towards the next phase: evaluation of serological assays for diagnostics and exposure assessment. medRxiv, 2020-042320077156 (2020). doi:10.1101/2020.04.23.20077156. Publisher: Cold Spring Harbor Laboratory Press. Accessed 2021-02-11

23. Van Elslande, J., Houben, E., Depypere, M., Brackenier, A., Desmet, S., André, E., Van Ranst, M., Lagrou, K., Vermeersch, P.: Diagnostic performance of seven rapid $\lg G / \lg M$ antibody tests and the Euroimmun $\lg A / \lg G$ ELISA in COVID-19 patients. Clinical Microbiology and Infection: The Official Publication of the European Society of Clinical Microbiology and Infectious Diseases 26(8), 1082-1087 (2020). doi:10.1016/j.cmi.2020.05.023

24. Neumann-Podczaska, A., Al-Saad, S.R., Karbowski, L.M., Chojnicki, M., Tobis, S., Wieczorowska-Tobis, K. COVID 19 - Clinical Picture in the Elderly Population: A Qualitative Systematic Review. Aging and Disease 11(4), 988-1008 (2020). doi:10.14336/AD.2020.0620. Accessed 2021-01-25

25. Öztaș Ayhan, H., Ișiksal, S.: Memory recall errors in retrospective surveys: A reverse record check study. Quality and Quantity 38(5), 475-493 (2005). doi:10.1007/s11135-005-2643-7. Accessed 2021-01-25

26. Graham, N.S.N., Junghans, C., Downes, R., Sendall, C., Lai, H., McKirdy, A., Elliott, P., Howard, R., Wingfield, D., Priestman, M., Ciechonska, M., Cameron, L., Storch, M., Crone, M.A., Freemont, P.S., Randell, P., McLaren, R., Lang, N., Ladhani, S., Sanderson, F., Sharp, D.J.: SARS-CoV-2 infection, clinical features and outcome of COVID-19 in United Kingdom nursing homes. The Journal of Infection 81(3), 411-419 (2020) doi:10.1016/j.jinf.2020.05.073

27. Magnavita, N., Tripepi, G., Di Prinzio, R.R.: Symptoms in Health Care Workers during the COVID-19 Epidemic. A Cross-Sectional Survey. International Journal of Environmental Research and Public Health 17(14), 5218 (2020). doi:10.3390/ijerph17145218. Number: 14 Publisher: Multidisciplinary Digital Publishing Institute. Accessed 2021-01-26

28. Rudberg, A.-S., Havervall, S., Månberg, A., Jernbom Falk, A., Aguilera, K., Ng, H., Gabrielsson, L., Salomonsson, A.-C., Hanke, L., Murrell, B., Mclnerney, G., Olofsson, J., Andersson, E., Hellström, C., Bayati, S., Bergström, S., Pin, E., Sjöberg, R., Tegel, H., Hedhammar, M., Phillipson, M., Nilsson, P., Hober, S., Thålin, C.: SARS-CoV-2 exposure, symptoms and seroprevalence in healthcare workers in Sweden. Nature Communications 11(1), 5064 (2020). doi:10.1038/s41467-020-18848-0. Number: 1 Publisher: Nature Publishing Group. Accessed 2021-01-26

29. Parente-Arias, P., Barreira-Fernandez, P., Quintana-Sanjuas, A., Patiño-Castiñeira, B.: Recovery rate and factors associated with smell and taste disruption in patients with coronavirus disease 2019. American Journal of Otolaryngology (2020). doi:10.1016/j.amjoto.2020.102648. Accessed 2021-02-22

30. Vaira, L.A., Deiana, G., Fois, A.G., Pirina, P., Madeddu, G., De Vito, A., Babudieri, S., Petrocelli, M., Serra, A., Bussu, F., Ligas, E., Salzano, G., De Riu, G.: Objective evaluation of anosmia and ageusia in COVID-19 patients: Single-center experience on 72 cases. Head \& Neck 42(6), 1252-1258 (2020). doi:10.1002/hed.26204 Accessed 2021-02-22

31. Schiffman, S.S.: Taste and smell losses in normal aging and disease. JAMA 278(16), 1357-1362 (1997)

32. Carfi, A., Bernabei, R., Landi, F., for the Gemelli Against COVID-19 Post-Acute Care Study Group: Persistent Symptoms in Patients After Acute COVID-19. JAMA 324(6), 603 (2020). doi:10.1001/jama.2020.12603. Accessed 2021-01-31

33. Huang, C., Huang, L., Wang, Y., Li, X., Ren, L., Gu, X., Kang, L., Guo, L., Liu, M., Zhou, X., Luo, J., Huang, Z., Tu, S., Zhao, Y., Chen, L., Xu, D., Li, Y., Li, C., Peng, L., Li, Y., Xie, W., Cui, D., Shang, L., Fan, G., Xu, J., Wang, G., Wang, Y., Zhong, J., Wang, C., Wang, J., Zhang, D., Cao, B.: 6-month consequences of COVID-19 in patients discharged from hospital: a cohort study. The Lancet 397(10270), 220-232 (2021). doi:10.1016/S0140-6736(20)32656-8. Publisher: Elsevier. Accessed 2021-01-31

34. Lam, M.H.-B., Wing, Y.-K., Yu, M.W.-M., Leung, C.-M., Ma, R.C.W., Kong, A.P.S., So, W.Y., Fong, S.Y.-Y., Lam, S.-P.: Mental morbidities and chronic fatigue in severe acute respiratory syndrome survivors: long-term follow-up. Archives of Internal Medicine 169(22), 2142-2147 (2009). doi:10.1001/archinternmed.2009.384 
35. Stavem, K., Ghanima, W., Olsen, M.K., Gilboe, H.M., Einvik, G.: Persistent symptoms 1.5-6 months after COVID-19 in non-hospitalised subjects: a population-based cohort study. Thorax (2020).

doi:10.1136/thoraxjnl-2020-216377. Publisher: BMJ Publishing Group Ltd Section: Brief communication. Accessed 2021-02-01

36. Schmier, J.K., Halpern, M.T.: Patient recall and recall bias of health state and health status. Expert Review of Pharmacoeconomics \& Outcomes Research 4(2), 159-163 (2004). doi:10.1586/14737167.4.2.159. Publisher:

Taylor \& Francis_eprint: https://doi.org/10.1586/14737167.4.2.159. Accessed 2021-02-19 
Figures and Tables

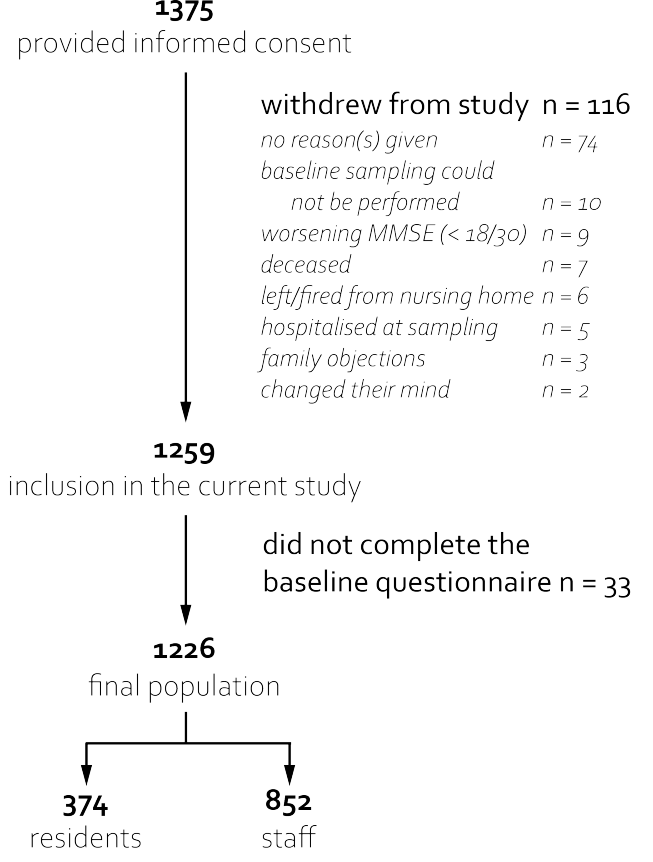

Figure 1: Flow chart of the study population. 1,359 participants of nursing homes provided us with an informed consent. Reasons for withdrawal are provided for the 116 drop-outs. 33 participants did not complete the baseline questionnaire, resulting in a final study population of 1,226 with 374 nursing home residents and 852 staff members. 


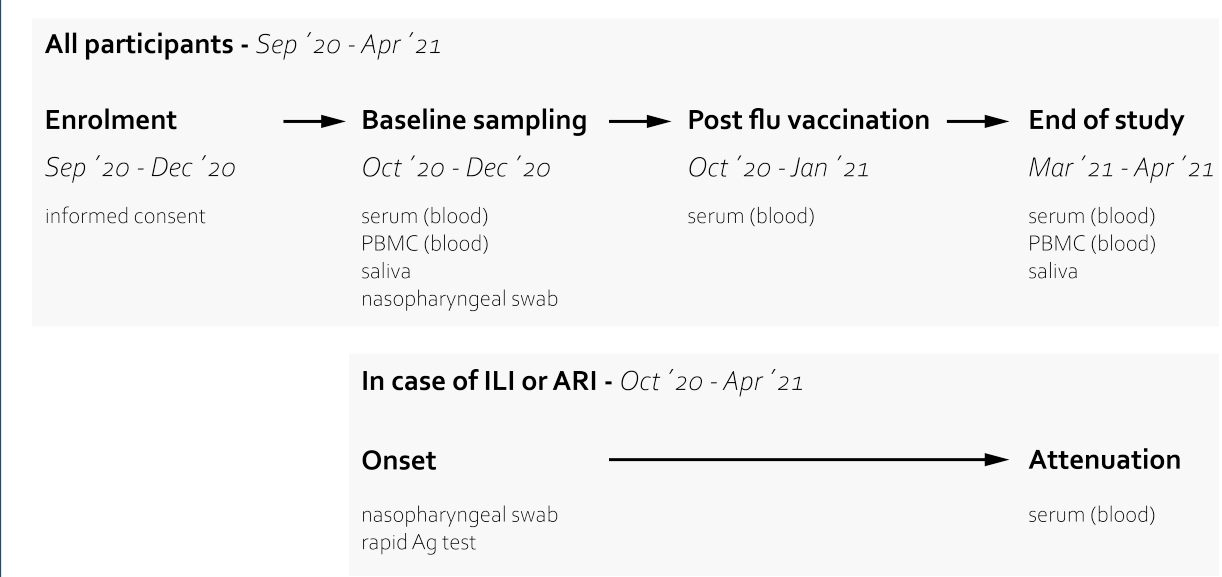

Figure 2: Flow of the PICOV study. Recruitment of the participants finished on December $8^{\text {th }}, 2020$, as did the baseline sampling (collection of a nasopharyngeal swab, saliva, serum blood, and heparinised blood for peripheral blood mononuclear cell [PBMC] isolation). Follow-up sampling after flu vaccination concluded on the $22^{\text {nd }}$ of January (collection of serum blood). Sampling at the end of the study is planned for spring 2021 (collection of saliva, serum blood, and heparinised blood for PBMC isolation). If a participant develops an influenzalike illness (ILI) or an acute respiratory infection (ARI), a nasopharyngeal swab is collected at the onset of symptoms to distinguish between a SARS-CoV-2 or influenza infection. After attenuations of the illness, approximately two to three weeks after the onset of symptoms, serum, and heparinised blood is collected. 


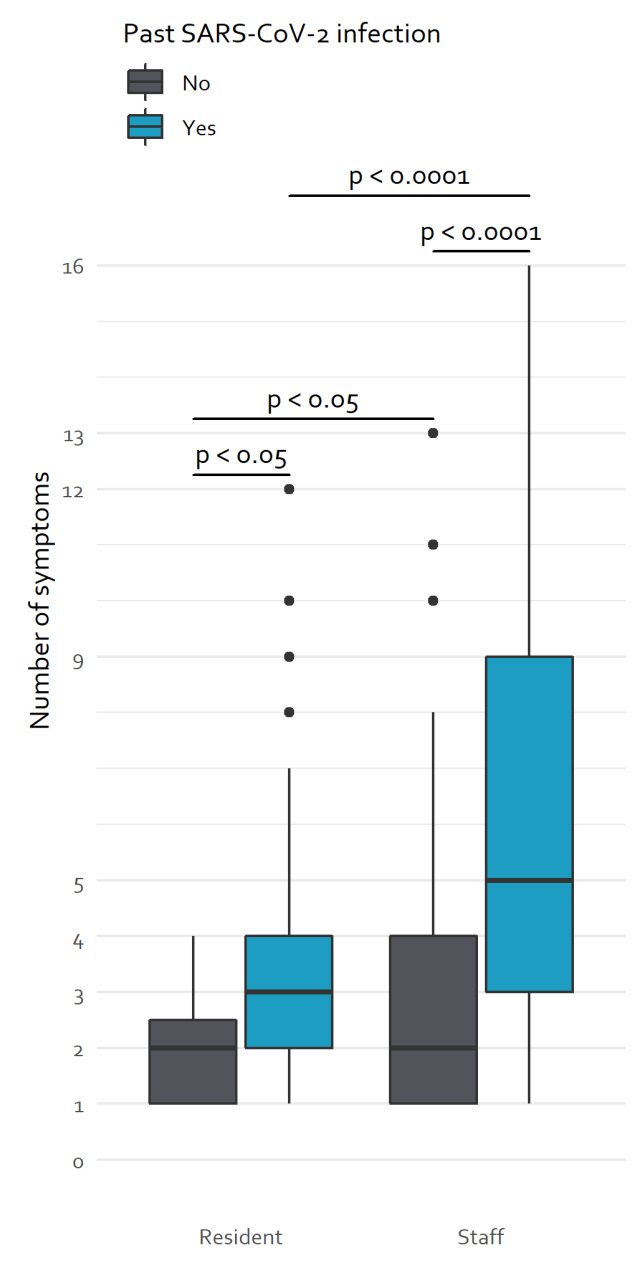

Figure 3: Number of symptoms in staff members and residents of nursing homes. Residents reported an average of $3.1( \pm 2.4)$ symptoms during a past SARS-CoV-2 infection, compared to $1.9( \pm 1.1)$ symptoms for residents without a previous infection. For staff members with a past SARS-CoV-2 infection, the number of symptoms averaged at $6.1( \pm 4.0)$ while those without an infection averaged $3.1( \pm 2.2)$ symptoms. 
Table 1: Sociodemographic characteristics of the total study population $(n=1,226)$, the staff members $(n=852)$, and the residents $(n=374)$. Data is presented as mean (SD) or $n(\%)$

\begin{tabular}{|c|c|c|c|}
\hline & Resident $(n=374)$ & Staff $(n=852)$ & Total $(n=1,226)$ \\
\hline Age, years & $81.9(10.7)$ & $44.2(11.5)$ & $55.7(20.7)$ \\
\hline Weight ${ }^{1}, \mathrm{~kg}$ & $70.4(15.6)$ & $72.9(14.4)$ & $72.1(14.9)$ \\
\hline Height $^{2}, \mathrm{~cm}$ & $166.0(9.2)$ & $167.0(8.2)$ & $166.9(8.5)$ \\
\hline $\mathrm{BMI}^{3}, \mathrm{~kg} / \mathrm{m}^{2}$ & $25.5(5.3)$ & $26.1(4.8)$ & $26.0(4.9)$ \\
\hline \multicolumn{4}{|l|}{ Gender } \\
\hline Male & $134(35.8 \%)$ & $125(14.7 \%)$ & $259(21.1 \%)$ \\
\hline Female & $240(64.2 \%)$ & $727(85.3 \%)$ & $967(78.9 \%)$ \\
\hline \multicolumn{4}{|l|}{ Ethnicity } \\
\hline European & $371(99.2 \%)$ & $747(87.7 \%)$ & $1,118(91.2 \%)$ \\
\hline Sub-Saharan Africa & $3(0.8 \%)$ & $36(4.2 \%)$ & $39(3.2 \%)$ \\
\hline North-African & $0(0.0 \%)$ & $21(2.5 \%)$ & $21(1.7 \%)$ \\
\hline Asian & $0(0.0 \%)$ & $24(2.8 \%)$ & $24(2.0 \%)$ \\
\hline Latin & $0(0.0 \%)$ & $8(1.0 \%)$ & $8(0.7 \%)$ \\
\hline Mixed & $0(0.0 \%)$ & $9(1.1 \%)$ & $9(0.7 \%)$ \\
\hline Unknown & $0(0.0 \%)$ & $7(0.8 \%)$ & $7(0.6 \%)$ \\
\hline \multicolumn{4}{|l|}{ Smoke } \\
\hline Current smoker & $48(12.8 \%)$ & $162(19.0 \%)$ & $210(17.1 \%)$ \\
\hline Past smoker & $56(15.0 \%)$ & $71(8.3 \%)$ & $127(10.4 \%)$ \\
\hline Non-smoker & $270(72.2 \%)$ & $619(72.7 \%)$ & $889(72.5 \%)$ \\
\hline \multicolumn{4}{|l|}{ Physical activity per day } \\
\hline None & $18(5.2 \%)$ & $72(8.6 \%)$ & $96(7.7 \%)$ \\
\hline Less than 30 minutes & $151(43.5 \%)$ & $135(16.8 \%)$ & $315(25.7 \%)$ \\
\hline $30-60$ minutes & $133(38.3 \%)$ & $219(27.3 \%)$ & $376(30.7 \%)$ \\
\hline At least 60 minutes & $45(13.0 \%)$ & $376(46.9 \%)$ & $439(35.8 \%)$ \\
\hline
\end{tabular}

Table 2: Past SARS-CoV-2 infection and seroprevalence at baseline sampling for the total population $(n=1,226)$, staff $(n=852)$, and residents $(n=374)$. The SARS-CoV-2 RT-qPCR results between March and August 2020 were self-reported at enrolment. Seroprevalence was assessed at baseline sampling.

\begin{tabular}{llrrr}
\hline Prior RT-qPCR result & Baseline Serology & $\begin{array}{r}\text { Resident } \\
(\mathrm{n}=374)\end{array}$ & $\begin{array}{r}\text { Staff } \\
(\mathrm{n}=852)\end{array}$ & $\begin{array}{r}\text { Total } \\
(\mathrm{n}=1,226)\end{array}$ \\
\hline Positive & Positive & $93(25.1 \%)$ & $304(35.6 \%)$ & $397(32.4 \%)$ \\
Positive & Negative & $19(5.1 \%)$ & $42(4.9 \%)$ & $61(4.9 \%)$ \\
Negative & Positive & $76(20.5 \%)$ & $106(12.4 \%)$ & $182(14.8 \%)$ \\
Negative & Negative & $183(49.3 \%)$ & $403(49.3 \%)$ & $586(47.8 \%)$ \\
\hline
\end{tabular}


Table 3: Profession of the staff members at the nursing home. Data is available for 849 staff members.

\begin{tabular}{lr}
\hline Profession & Staff $(\mathrm{n}=849)$ \\
\hline Medical & $11(1.3 \%)$ \\
Medical Doctor & $7(0.8 \%)$ \\
Not specified & $4(0.5 \%)$ \\
Nurse & $198(23.2 \%)$ \\
Paramedical & $355(41.7 \%)$ \\
Nursing assistant & $253(29.7 \%)$ \\
Physical therapist & $48(5.6 \%)$ \\
Occupational therapist & $37(4.3 \%)$ \\
Logopaedics & $3(0.4 \%)$ \\
Psychologist & $5(0.6 \%)$ \\
Not specified & $9(1.1 \%)$ \\
Non-medical & $258(30.3 \%)$ \\
Administration & $87(10.2 \%)$ \\
Support staff & $97(11.4 \%)$ \\
Kitchen staff & $37(4.3 \%)$ \\
Animators & $18(2.1 \%)$ \\
Social worker & $2(0.2 \%)$ \\
Not specified & $17(2.0 \%)$ \\
COVID department & $27(3.2 \%)$ \\
\hline
\end{tabular}

Table 4: Self-reported symptoms the 640 'past SARS-CoV-2 infection' participants experienced from March $1^{\text {st }}$ to August $31^{\text {st }} 2020$ during their COVID-19 episode. Chi-squared statistics $\left(\chi^{2}\right)$ was used to compare the residents with the staff members.

\begin{tabular}{lrrrr}
\hline Symptom & $\begin{array}{r}\text { Resident } \\
(\mathrm{n}=188)\end{array}$ & $\begin{array}{r}\text { Staff } \\
(\mathrm{n}=452)\end{array}$ & $\begin{array}{r}\text { Total } \\
(\mathrm{n}=640)\end{array}$ & $\chi^{2}$ p-value \\
\hline No symptoms & $111(59 \%)$ & $127(28.1 \%)$ & $238(37.2 \%)$ & $<0.0001$ \\
Fatigue & $40(21.3 \%)$ & $256(56.6 \%)$ & $296(46.2 \%)$ & $<0.0001$ \\
Ageusia and/or anosmia & $12(6.4 \%)$ & $202(44.7 \%)$ & $214(33.4 \%)$ & $<0.0001$ \\
Headache & $7(3.7 \%)$ & $195(43.1 \%)$ & $202(31.6 \%)$ & $<0.0001$ \\
Cough & $34(18.1 \%)$ & $149(33.0 \%)$ & $183(28.6 \%)$ & $<0.0001$ \\
Dyspnea & $30(16.0 \%)$ & $148(32.7 \%)$ & $178(27.8 \%)$ & $<0.0001$ \\
Fever & $39(20.7 \%)$ & $141(31.2 \%)$ & $180(28.1 \%)$ & 0.0098 \\
Pain (any) & $11(5.9 \%)$ & $120(26.5 \%)$ & $131(20.5 \%)$ & $<0.0001$ \\
Muscle pain & $5(2.7 \%)$ & $99(21.9 \%)$ & $104(16.2 \%)$ & $<0.0001$ \\
Joint pain & $2(1.1 \%)$ & $76(16.8 \%)$ & $78(12.2 \%)$ & $<0.0001$ \\
Chest pain & $2(1.1 \%)$ & $53(11.7 \%)$ & $55(8.6 \%)$ & $<0.0001$ \\
Abdominal pain & $1(0.5 \%)$ & $16(3.5 \%)$ & $17(2.7 \%)$ & 0.06 \\
Shivering & $4(2.1 \%)$ & $118(26.1 \%)$ & $122(19.1 \%)$ & $<0.0001$ \\
Sore throat & $5(2.7 \%)$ & $116(25.7 \%)$ & $121(18.9 \%)$ & $<0.0001$ \\
Rhinitis & $7(3.7 \%)$ & $87(19.2 \%)$ & $94(14.7 \%)$ & $<0.0001$ \\
Diarrhoea & $13(6.9 \%)$ & $70(15.5 \%)$ & $83(13.0 \%)$ & 0.0049 \\
Nausea & $7(3.7 \%)$ & $46(10.2 \%)$ & $53(8.3 \%)$ & 0.0111 \\
Confused & $7(3.7 \%)$ & $23(5.1 \%)$ & $30(4.7 \%)$ & 0.59 \\
Anorexia & $6(3.2 \%)$ & $22(4.9 \%)$ & $28(4.4 \%)$ & 0.46 \\
Worsening prior & $4(2.1 \%)$ & $11(2.4 \%)$ & $15(2.3 \%)$ & 1.00 \\
respiratory problems & $0(0.0 \%)$ & $10(2.2 \%)$ & $10(1.6 \%)$ & 0.09 \\
Rash & & & & \\
\hline
\end{tabular}


Table 5: Self-reported symptoms the 586 'no past SARS-CoV-2 infection' participants experienced March $1^{\text {st }}$ to August $31^{\text {st }} 2020$. Chi-squared statistics $\left(\chi^{2}\right)$ was used to compare the residents with the staff members.

\begin{tabular}{lrrrr}
\hline Symptom & $\begin{array}{r}\text { Resident } \\
(\mathrm{n}=183)\end{array}$ & $\begin{array}{r}\text { Staff } \\
(\mathrm{n}=403)\end{array}$ & $\begin{array}{r}\text { Total } \\
(\mathrm{n}=586)\end{array}$ & $\chi^{2}$ p-value \\
\hline No symptoms & $161(88.0 \%)$ & $291(72.2 \%)$ & $452(77.1 \%)$ & $<0.0001$ \\
Fatigue & $4(2.2 \%)$ & $50(12.4 \%)$ & $54(9.2 \%)$ & $<0.0001$ \\
Ageusia and/or anosmia & $0(0.0 \%)$ & $3(0.7 \%)$ & $3(0.5 \%)$ & 0.59 \\
Headache & $1(0.5 \%)$ & $56(13.9 \%)$ & $57(9.7 \%)$ & $<0.0001$ \\
Dyspnea & $3(1.6 \%)$ & $21(5.2 \%)$ & $24(4.1 \%)$ & 0.07 \\
Cough & $7(3.8 \%)$ & $30(7.4 \%)$ & $37(6.3 \%)$ & 0.14 \\
Fever & $2(1.1 \%)$ & $17(4.2 \%)$ & $19(3.2 \%)$ & 0.08 \\
Pain (any) & $2(1.1 \%)$ & $5(1.2 \%)$ & $7(1.2 \%)$ & 1.00 \\
Muscle pain & $1(0.5 \%)$ & $4(1.0 \%)$ & $5(0.9 \%)$ & 0.95 \\
Joint pain & $1(0.5 \%)$ & $4(1 \%)$ & $5(0.9 \%)$ & 0.95 \\
Chest pain & $0(0.0 \%)$ & $2(0.5 \%)$ & $2(0.3 \%)$ & 0.85 \\
Abdominal pain & $0(0.0 \%)$ & $1(0.2 \%)$ & $1(0.2 \%)$ & 1.00 \\
Shivering & $0(0.0 \%)$ & $21(5.2 \%)$ & $21(3.6 \%)$ & 0.0037 \\
Sore throat & $5(2.7 \%)$ & $52(12.9 \%)$ & $57(9.7 \%)$ & 0.0002 \\
Diarrhoea & $4(2.2 \%)$ & $22(5.5 \%)$ & $26(4.4 \%)$ & 0.12 \\
Rhinitis & $4(2.2 \%)$ & $38(9.4 \%)$ & $42(7.2 \%)$ & 0.0029 \\
Nausea & $1(0.5 \%)$ & $11(2.7 \%)$ & $12(2.0 \%)$ & 0.16 \\
Anorexia & $1(0.5 \%)$ & $0(0.0 \%)$ & $1(0.2 \%)$ & 0.69 \\
Confused & $0(0.0 \%)$ & $4(1.0 \%)$ & $4(0.7 \%)$ & 0.42 \\
Worsening prior & $0(0.0 \%)$ & $2(0.5 \%)$ & $2(0.3 \%)$ & 0.85 \\
respiratory problems & $0(0.0 \%)$ & $5(1.2 \%)$ & $5(0.9 \%)$ & 0.30 \\
Rash & & & & \\
\hline
\end{tabular}

Table 6: Persisting self-reported symptoms of the 94 'past SARSCoV-2 infection' participants experienced at baseline sampling. Chisquared statistics $\left(\chi^{2}\right)$ was used to compare the residents with the staff members

\begin{tabular}{lrrrr}
\hline Symptom & $\begin{array}{r}\text { Resident } \\
(\mathrm{n}=8)\end{array}$ & $\begin{array}{r}\text { Staff } \\
(\mathrm{n}=86)\end{array}$ & $\begin{array}{r}\text { Total } \\
(\mathrm{n}=94)\end{array}$ & $\chi^{2}$ p-value \\
\hline Fatigue & $2(25.0 \%)$ & $48(55.8 \%)$ & $50(53.2 \%)$ & $<0.0001$ \\
Ageusia and/or anosmia & $1(12.5 \%)$ & $34(39.5 \%)$ & $35(37.2 \%)$ & $<0.0001$ \\
Headache & $0(0.0 \%)$ & $13(15.1 \%)$ & $13(13.8 \%)$ & $<0.0001$ \\
Dyspnea & $2(25.0 \%)$ & $31(36.0 \%)$ & $33(35.1 \%)$ & $<0.0001$ \\
Cough & $4(50.0 \%)$ & $4(4.7 \%)$ & $8(8.5 \%)$ & $<0.0001$ \\
Pain (any) & $0(0.0 \%)$ & $11(12.8 \%)$ & $11(11.7 \%)$ & $<0.0001$ \\
Sore throat & $0(0.0 \%)$ & $6(7.0 \%)$ & $6(6.4 \%)$ & $<0.0001$ \\
Diarrhoea & $0(0.0 \%)$ & $2(2.3 \%)$ & $2(2.1 \%)$ & 0.16 \\
Rhinitis & $0(0.0 \%)$ & $3(3.5 \%)$ & $3(3.2 \%)$ & 0.046 \\
Nausea & $0(0.0 \%)$ & $1(1.2 \%)$ & $1(1.1 \%)$ & 0.23 \\
Anorexia & $1(12.5 \%)$ & $1(1.2 \%)$ & $2(2.1 \%)$ & $<0.0001$ \\
Confused & $0(0.0 \%)$ & $8(9.3 \%)$ & $8(8.5 \%)$ & $<0.0001$ \\
Worsening prior & $0(0.0 \%)$ & $5(5.8 \%)$ & $5(5.3 \%)$ & 0.025 \\
respiratory problems & $0(0.0 \%)$ & $2(2.3 \%)$ & $2(2.1 \%)$ & 0.16 \\
Rash & & & & \\
\hline
\end{tabular}




\section{Figures}

\section{5}

provided informed consent

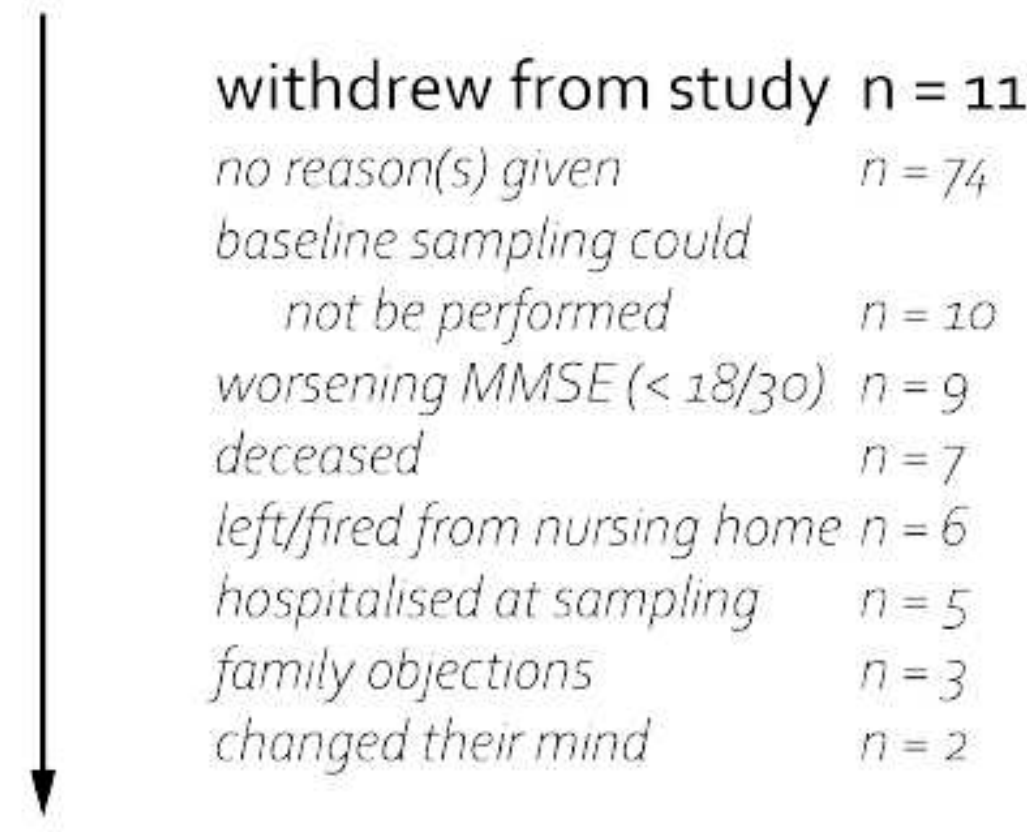

\section{9}

inclusion in the current study

\section{did not complete the baseline questionnaire $n=33$}

1226

final population



\section{Figure 1}

Flow chart of the study population. 1,359 participants of nursing homes provided us with an informed consent. Reasons for withdrawal are provided for the 116 drop-outs. 33 participants did not complete the 
baseline questionnaire, resulting in a final study population of 1,226 with 374 nursing home residents and 852 staff members.
All participants - Sep '2O-Apr' 21

\section{Enrolment}

Sep '20-Dec'20

informed consent

\section{$\longrightarrow$ Baseline sampling}

Oct'20-Dec'20

serum (blood)

PBMC (blood)

saliva

nasopharyngeal swab $\longrightarrow$ Post flu vaccination

Oct'20 - Jan' 21

serum (blood)
PBMC (blood)

saliva

In case of ILI or ARI - Oct' 20 - Apr' $21^{\prime}$

Onset

nasopharyngeal swab

rapid Ag test
Attenuation

serum (blood)

\section{Figure 2}

Flow of the PICOV study. Recruitment of the participants finished on December 8th, 2020, as did the baseline sampling (collection of a nasopha-ryngeal swab, saliva, serum blood, and heparinised blood for peripheral blood mononuclear cell [PBMC] isolation). Follow-up sampling after flu vaccination concluded on the 22nd of January (collection of serum blood). Sampling at the end of the study is planned for spring 2021 (collection of saliva, serum blood, and heparinised blood for PBMC isolation). If a participant develops an influenza-like illness (ILI) or an acute respiratory infection (ARI), a nasopharyngeal swab is collected at the onset of symptoms to distinguish between a SARS-CoV-2 or influenza infection. After attenuations of the illness, approximately two to three weeks after the onset of symptoms, serum, and heparinised blood is collected. 


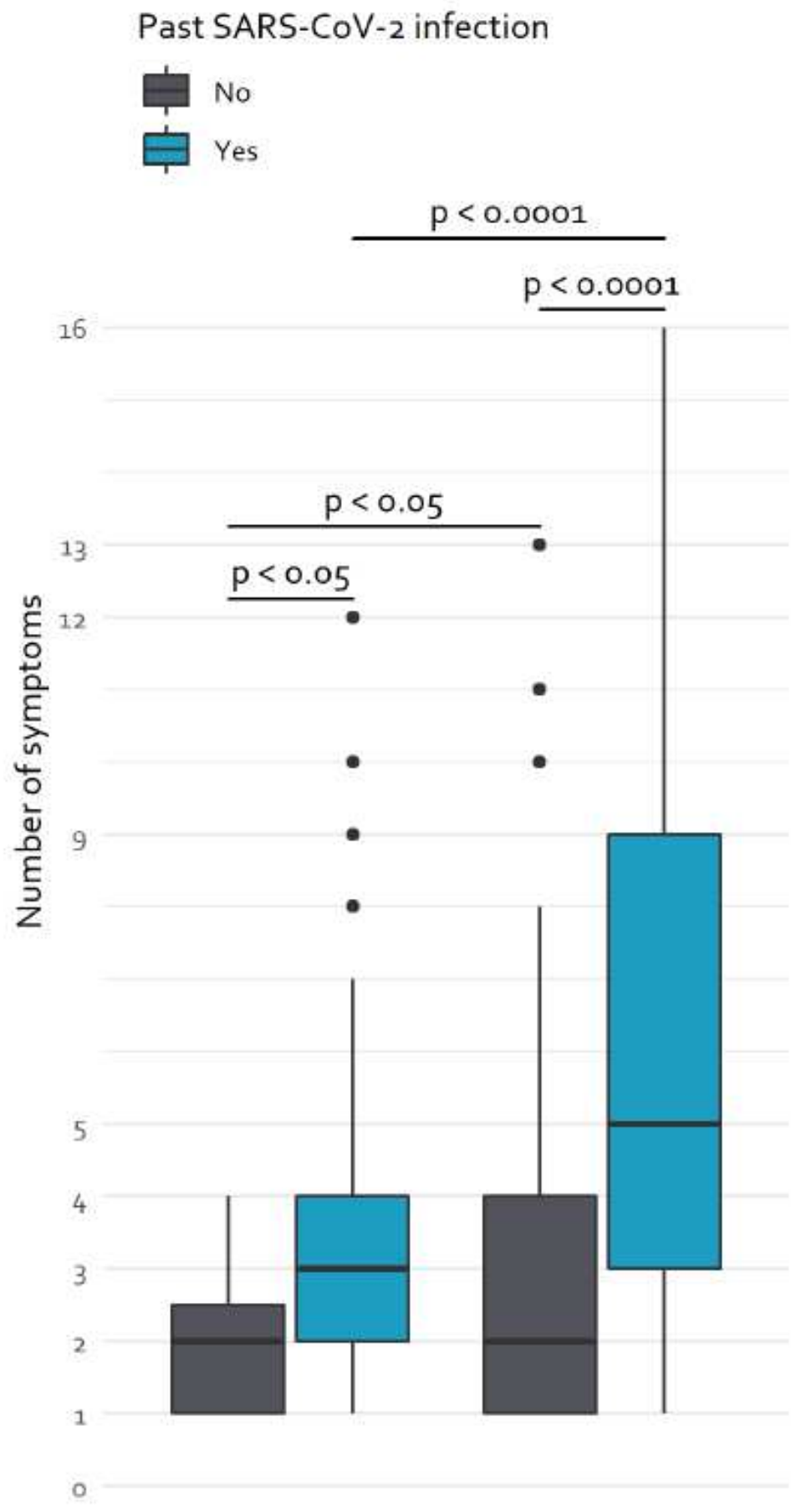

Resident

Staff

\section{Figure 3}

Number of symptoms in staff members and residents of nurs-ing homes. Residents reported an average of $3.1( \pm 2.4)$ symptoms during a past SARS-CoV-2 infection, compared to $1.9( \pm 1.1)$ symptoms for residents without a previous infection. For staff members with a past SARS-CoV-2 infection, the number of symptoms averaged at $6.1( \pm 4.0)$ while those without an infection averaged $3.1( \pm 2.2)$ symptoms. 